\title{
NATURAL HISTORY OF MULTIPLE SCLEROSIS
}

G C Ebers

\begin{abstract}
7 he natural history of multiple sclerosis (MS) should be considered in the context of better understood disorders where the problems of definition, ascertainment, and outcomes can be more easily appreciated.
\end{abstract}

\section{DEFINITION OF NATURAL HISTORY}

The definition of the natural history of acute illnesses has been difficult to establish. Imagine, however, the degree of uncertainty in an earlier age when fever itself was considered to be a disease. The appearance of fever could represent a banal upper respiratory disease or underlying untreatable infection or malignancy. Good outcomes offered unlimited opportunities for unwarranted claims for credit. Bad outcomes were blamed on "sin", and the physician's contribution would be one of either commission or omission. When evaluation consisted largely of taking the pulse and measuring the temperature, the study of physical findings was exhaustive. Text books of thermometry described undoubtedly accurate vagaries of fever long forgotten in diseases still commonly seen today. ${ }^{1}$ Fever became rationally dissected into a large number of specific entities with the development of microbiology changing the focus of diagnosis and prognosis to the specific clinical features characterising each causal organism. It must have been both surprising and disappointing to find that a large variability in outcome remained, even when the microbiological cause was pinpointed. The spectrum of outcome for many disorders ranged from self limited nuisance to fatality (for example, pulmonary tuberculosis infection) and was hardly narrower than for the "diagnosis" of fever. The failure of outcome to define disease entity had to be repeatedly demonstrated. Only recently has attention shifted significantly to the study of host factors and in the last generation have tools become available to investigate this with any power.

The problems of defining outcome seen in the infectious disorders are compounded in the more chronic diseases where, in most, survival is excellent in the short term. The dearth of information for most chronic diseases is shocking to the present day "internet generation" of the newly diagnosed who find themselves frequently unable to find answers to the simple questions of what will likely happen to them. The stock of studies of natural history in common diseases is small, and however surprising the scarcity of such information for any disease might be, it is understandable when the reasons are considered.

Inception cohorts are often impossible for diseases like MS where clinical onset is often discordant with biological onset and the duration of the disease easily surpassing the innate domiciliary inertia of investigators if not their lifespan. Untreated patients have become scarce. This by no means precludes the collection of potentially useful data in circumstances where effectiveness of treatment (as opposed to efficacy) has not been established.

There can be few diseases in which these considerations are more relevant than in MS. The disorder usually spans some four to five decades in duration, posing particular difficulty for treatment evaluation. ${ }^{2}$ One of my teachers, commenting on the value of a surgical procedure, which he had devised, stated that clinical trials were unnecessary since it resembled the suspension of a lead ball out the window-if it went up when released you knew this was significant. In the context of a clinical round such a statement provided predictable mirth among medical colleagues. However, with hindsight there is some truth to his statement in so far as treatment of disease is concerned and there is more depth to his anti-gravitational metaphor than there might seem. The expectations of clinical outcome are often reminiscent of the plausible, but erroneous, expectation that heavier objects fall more quickly than lighter ones. The placebo arms of unblinded trials with unblinded examiners are rated worse than are their ineffectively treated counterparts ${ }^{3}$ but generally fare better than they were expected to do. However, hospital based patients do worse more rapidly than those that are clinic based who in turn tend to do worse than do those in epidemiological samples. 
Bradford Hill in his seminal discussion on the landmark UK Medical Research Council streptomycin trial in tuberculosis pointed out that a randomised clinical trial is not needed where the outcome is certain as in the case of tuberculous meningitis. Certainty in variable diseases may not be completely unattainable or at least if one can abide near certainty. In long term diseases this is a function of uniformity of ascertainment, the outcome measure used, and the patient-years of observation which itself may in the long run supersede some of the vagaries of ascertainment (see below). It can hardly be argued that late outcome patient years increase in unit value (the 10 year data in 10 patients may be more valuable than six month data in 200). It remains to be shown how this might be quantitated and represents a more useful goal than the recent enthusiasm for finding more sensitive short term outcome measures in a disease awash with Fleiss type Ib errors. ${ }^{4}$

There are numerous lessons to be derived from natural history but most of them can be conveniently divided into those that:

- illuminate the pathogenesis of disease

- relate to the practical exercise of prognostication and

- relate to the evaluation of effectiveness in the context of treatment.

\section{Pathogenesis of MS}

Consideration of pathogenesis is beyond the scope of this review but it is widely believed that MS is an autoimmune disorder, mediated by $\mathrm{T}$ cells. The importance of the loss of axons in the production of unremitting disability has been increasingly appreciated. Recent studies have suggested pathological heterogeneity and the presence of several distinct phenotypes. The presence of heterogeneity and complexity is the rule in multifactorial disorders, so descriptions based on the phenotype of what we now call MS may well fragment in the future into several distinct disorders. However attractive in concept, attempts to subdivide the disease based on clinical phenomenology have met with limited success. Nevertheless both the study of natural history and the results of therapeutic trials have shed unexpected light on the relation between relapse and progression.

\section{Long term outcome in the London, Ontario cohort}

The population from which many of the conclusions in this study are derived consists of more than 1000 patients from a geographic base followed for a mean of 25 years. More than $95 \%$ were thought to have MS verified by their subsequent clinical course and fewer than $5 \%$ were lost to follow up before reaching Disability Status Scale (DSS) levels (Kurtzke) of 6, 8, and 10.5 This cohort has been the subject of a series of publications and several others in preparation. In this review we draw heavily on this material. The data have limitations. Patients were seen at yearly intervals although more often in the first two years. Exacerbation rates were later then derived from yearly visits and results will be an underestimate although the Middlesex County subcohort (for whom the MS clinic delivered near primary care) provides an independent "reality check". Transitions from one level of disability to another were left blank if undocumented; however, this had little effect in practice since later levels were available.

\section{Survival in MS}

The survival in MS is shortened but overall it is surprisingly little affected in western countries. Compared to the general population (which lives less long than the insured population), MS patients live some 5-7 years less, probably similar to the reduction in life expectancy attributable to smoking one pack of cigarettes a day. In some populations, suicide is a significant factor in the reduction in survival. There does appear to be an effect of co-morbid disease only insofar as they result from the consequences of MS itself. Cancer and atherosclerosis are decreased if anything but this may reflect ascertainment. The question of co-occurrence of autoimmune disease is less clear but, uveitis aside, if there is any increase over the rate corrected for similar northern European populations, it must be slight as numerous studies have shown conflicting results. The long survival in MS is not unrelated to the difficulty in studying its natural history.

\section{Exacerbations and relapses}

These are certainly the most florid feature of this disease and a typical relapse in the appropriate anatomical location is often sufficient for an experienced clinician to make the diagnosis of MS with considerable reliability. Nevertheless, the relation between relapse and outcome has not been clarified. It is widely believed that disability in MS results from a series of successive exacerbations, each adding to a growing accumulation of deficits. To be sure every clinician harbours unquestioned anecdotes in which acute relapses at the beginning or during the course of the disease leave patients with little or no recovery. In such instances, relapses may carry a major burden. However, overall such events are unusual, with the exception of the relatively rare subcategory of MS known as Devic's disease where deterioration is largely in a stepwise fashion associated with successive exacerbations. Furthermore, it must be remembered that any such effect would be washed out as higher levels of disability supervene unless there was a direct influence on what happens later. In Japan the oriental form of MS is Devic-like and deterioration is stepwise with relapse-free progression being the exception.

On the other side of the relapse question are patients with single or no relapses (as in single attack progressive or primary progressive MS) where steady deterioration dominates the disease and single or rare relapses subsequent to years of progression have trivial impact on outcome. It has become commonplace in recent years to adapt McAlpine's original schematic ${ }^{6}$ to indicate that relapses leave a succession of residual deficits, following which the chronic progressive phase supervenes with a takeoff point in midscale. Re-inspection of his original graphs is warranted.

It is not surprising given these observations that, even in the minds of experienced clinicians, the relation between relapses and long term outcome is uncertain. This is a situation in which detailed analysis of a natural history database can yield interesting answers bearing on both concepts of pathogenesis and on practical applications.

\section{Relapses and long term outcome}

At the time of our original studies, we examined the role of relapses and long term outcome and found a highly significant association between relapses in the first two years and shortened time to walking with a cane and using a wheelchair. ${ }^{7}$ It seemed that the association was strongest for those having four or more exacerbations (table 1). Causality was far from established and the possibility that early attacks 
Table 1 Median times to DSS 3 (moderate disability in one functional scale) and 6 (walks $100 \mathrm{~m}$ with aids) in a population based cohort for primary progressive MS and relapsing remitting MS derived from clinical onset of disease based on 25000 patient-years of observation in the London, Ontario cohort (to the nearest integer)

Time to disability (medians) first two year attack rate

- One attack: 13 years

- Two attacks: 8 years

- Three attacks: 9 years

- Four attacks: 8 years

- Five + attacks: 3 years

EDSS 6

- One attack: 20 years

- Two attacks: 17 years

- Three attacks: 18 years

- Four attacks: 14 years

- Five + attacks: 7 years

EDSS, Expanded Disability Status Scale (Kurtzke).

and disability were associated rather than causally related remained a viable option. With the advent of treatments, which could clearly prevent some relapses and their widespread usage, this became a question of practical relevance.

At the time of our original reports ${ }^{8-10}$ our long term follow up extended just beyond 10 years on average and in the year 2000 had extended out to 25 years with a total 25000 patient-years of observation. This allowed a more definitive examination of this relation. The longer data set confirmed the original result, indeed magnified it. However, there are difficulties in the much hoped for conclusion that relapses themselves caused long term deterioration and ergo suppression would lead in the end to the hoped for reward.

In the first instance, most of the effect on outcome from exacerbation in the first two years was contained in the first year. Since patients were only seen yearly, we were confident that our relapse figures would be an underestimate. Nevertheless there is some pragmatic value to them since they did take into account those for which patients sought medical attention. Analysis of these results does show that the development of the progressive course of the disease dwarfs all other predictive measures (Wingerchuk et al, unpublished data, 2001).

We have examined relapses in the pre-progressive phase, the progressive phase, and total relapses and find no relation between their frequency and outcome. The degree of recovery from the first exacerbation is not predictive so that those with no recovery do as well or as badly as those with complete recovery (Ebers et al, unpublished data, 2001). Parallelling the untoward effect of the number of exacerbations in the first year, we find that polysymptomatic onset has a modest predictive value with a relative risk of 2 for time to DSS 6 . These findings suggest that early frequent relapses are deleterious for long term outcome via an indirect mechanism or are simply a concomitant of what is destined to be a more rapid clinical course.

\section{Progression}

The development of a progressive course is by far the most deleterious event in the case of an MS patient. A multivariate analysis with relative risks of progression to cane requirement is given in the box. The box shows the dominant effect of this clinical feature. It is important to point out that the identification of the onset of this phase is not straightforward at the time it may seem to begin. Often the onset of progression is clouded by concomitant relapses, but it would be reasonable to expect a priori that such a degenerative process would begin long before it produces clinical symptoms, simply because of the known plasticity of the nervous system. In groups which are progressive from onset or in those that have had a single exacerbation outside the spinal cord and then subsequently become progressive, it can
Relative risks $(R R)$ for reaching DSS 6, 8, and 10

6 (walking $100 \mathrm{~m}$ with aids), 8 (wheelchair), and 10 (death from MS) in a multivariate analysis for selected predictors of outcome in the London, Ontario cohort (to the nearest integer).

(1) Progressive course: largest single predictor risk ratio $(R R=6)$

(2) Relapse rate: year 1/year $2(R R=3)$

(3) First interattack interval $(R R=2)$

(4) Polysymptomatic onset $(\mathrm{RR}=2)$

(5) Time to early disability $(R R=2)$

The "first interattack interval" is the time between the first and second attack. The "time to early disability" is the time from onset to EDSS 3

be shown that rate of progression is virtually identical. Surprisingly similar rates are to be found in those with secondary progressive MS irrespective of the number of preceding relapses.

\section{Primary progressive MS}

It would be easy to get the impression that disease phenotypes would be defined by the magnetic resonance imaging (MRI) appearance, if the situation in primary progressive MS is any indication. Based upon a study containing a modest number of patients and on the application of parametric statistics to non-parametric distributions of MRI lesions, separate disease status for this condition has been claimed. ${ }^{11}$ Its exact nosological location is uncertain but suggestions that many such patients have MRI criteria which are not fulfilling those described for relapse onset MS are not supported in the large study of more than 400 patients with primary progressive MS. ${ }^{12}$ We are sceptical that this represents anything more than MS without the relapsing remitting phase as suggested many years ago by Minderhoud. ${ }^{13}$ The disease course has been studied in detail by Cottrell et al, ${ }^{5}$ and the course of progression is not different from that seen in those with secondary progressive MS after multiple or single attacks (Kremenchutzky et al, unpublished data, 2001).

\section{Conclusions from studies of natural history in London, Ontario}

The descriptive results from a 25000 patient-year cohort will have practical applications to the assignation of prognosis and the planning and interpretation of clinical trials, and may serve as a virtual control for clinical trials in the future. However, they also shed some light on nosology and more importantly pathogenesis. As tempting as it may be to associate exacerbations with outcome, it seems likely that the apparent role of exacerbations early in the disease is more a reflection of the active state of the illness than having a direct causal relation to subsequent disability. Neither the total number of attacks nor the frequency before the onset of progression are related to the hard outcome measures of time to use of cane, bed or grave. Once the disease begins to develop a progressive phase, perhaps at a time long before clinical symptoms arise, the course of deterioration seems remarkably the same irrespective of the presence of prior or subsequent exacerbations or their frequency. This has implications for the likelihood that relapse suppression therapies will be effective when progression has begun. ${ }^{14}$ Confavreux first made this observation ${ }^{15}$ and our data support it strongly. The notion of primary progressive disease 
as a distinct disease entity is on shaky ground, supported by neither genetic epidemiology nor by clinical course. The outcomes for progressive disease in all forms seem remarkably similar when groups are compared.

\section{References}

1 Wunderlich's medical thermometry. London: New Sydenham Society, 1871.

2 Ebers GC. Chapter 6: The natural history of MS. In: Paty DW, Ebers GC. Multiple sclerosis. Philadelphia: FA Davis and Co, 1998.

3 Noseworthy JH, Ebers GC, Vandervoort MK, et al. The impact of blinding on the results of a randomised, placebo-controlled multiple sclerosis clinical trial. Neurology 1994;44:16-20.

4 Fleiss J. Statistical methods for rates and proportions. New York: John Wiley \& Sons, 1981.

5 Cottrell D, Kremenchutzky M, Rice GP, et al. The natural history of multiple sclerosis: a geographically based study. 5 . The clinical features and natural history of primary progressive multiple sclerosis. Brain 1999;122:625-39.

6 McAlpine D, Compston ND, Lumsden CE. Multiple sclerosis. Edinburgh: Livingstone, 1955.

7 Weinshenker BG, Bass B, Rice GPA, et al. The natural history of multiple sclerosis: a geographically based study. 2. Predictive value of the early clinical course. Brain 1989;112:1419-28.
8 Weinshenker BG, Bass B, Rice GPA, et al. Natural history of multiple sclerosis: a geographically based study. 1. Clinical course and disability Brain 1989;112:133-46.

9 Weinshenker BG, Bass B, Rice GPA, et al. The natural history of multiple sclerosis: a geographically based study. 3. Multivariate analysis of predictive factors and models of outcome. Brain 1991;114:1045-56.

10 Weinshenker BG, Bass B, Rice GPA, et al. The natural history of multiple sclerosis: a geographically based study. 4. Applications to planning and interpretation of clinical therapeutic trials. Brain 1991;114:1057-67.

11 Thompson AJ, Polman $\mathrm{CH}$, Miller $\mathrm{DH}$, et al. Primary progressive multiple sclerosis [Review]. Brain 1997;120:1085-96.

12 Kremenchutzky M, Lee D, Rice GP, et al. Diagnostic brain MRI findings in primary progressive multiple sclerosis. Multiple Sclerosis 2000;6: 81-5.

13 Minderhoud JM. On the pathogenesis of multiple sclerosis: a revised model of the cause(s) of multiple sclerosis, especially based on epidemiological data. Clin Neurol Neurosurg 1994;96:135-42.

14 Rice GP, Ebers GC. Interferons in the treatment of multiple sclerosis: do they prevent the progression of the disease? Arch Neurol 1998;55:1578-83.

15 Confavreux C. Relapses and progression of disability in multiple sclerosis. N Engl J Med 2000;343:1430-8.

\title{
7th European Forum on Quality Improvement in Health Care
}

\author{
21-23 March 2002 \\ Edinburgh, Scotland
}

We are delighted to announce this forthcoming conference in Edinburgh. Authors are invited to submit papers (call for papers closes on Friday 5 October 2001) and delegate enquiries are welcome.

The themes of the Forum are:

- Leadership, culture change, and change management

- Achieving radical improvement by redesigning care

- Health policy for lasting improvement in health care systems

- Patient safety

- Measurement for improvement, learning, and accountability

- Partnership with patients

- Professional quality: the foundation for improvement

- Continuous improvement in education and training

- People and improvement.

Presented to you by the BMJ Publishing Group (London, UK) and Institute for Healthcare Improvement (Boston, USA). For more information contact: quality@bma.org.uk or look at the website www.quality.bmjpg.com. Tel: +44 (0)20 7383 6409; fax: +44 (0)20 73736869 . 